\title{
Decision Support System in a Patient-Centered Medical Home
}

Kalyan S. Pasupathy ${ }^{1,4}$, Karl M. Kochendorfer ${ }^{2,4}$, Gordon D. Brown ${ }^{1,4}$, Lanis L. Hicks ${ }^{1,4}$, Linsey M. Barker ${ }^{3,4}$ and Ricky C. Leung ${ }^{1,4}$ ${ }^{1}$ Health Management \& Informatics $\quad{ }^{2}$ Family and Community Medicine $\quad{ }^{3}$ Industrial \& Manufacturing Systems Engineering University of Missouri

\begin{abstract}
Lack of sufficient primary care to manage chronic diseases has been quoted as a major drawback of the healthcare system within the United States. Patient-Centered Medical Home is a care delivery model to transform how primary care is delivered. The information technology revolution has brought about several advancements and solutions for medicine and care delivery, and medical homes are no exception to this. However, it is only through a robust decision support system that these medical homes can in fact provide truly coordinated and patient-centered care. The paper describes preliminary work that has been completed at the University of Missouri Health System and next steps in achieving high quality care delivery through a decision support system implementation.

\section{Introduction and Background}

An estimated 100 million Americans have at least one chronic condition, and half of these with more than one [1]. Improving Chronic Illness Care (ICIC) identifies these deficiencies in chronic care - rushed practitioners not following established practice guidelines, lack of care coordination, lack of active follow-up to ensure the best outcomes, and patients inadequately trained to manage their illnesses [2]. Medical homes change the provision of care from encounter-centric to patient-centric, based on principles of the chronic care model, a framework for improving chronic disease care through care coordination, active follow-up for self-management, clinical decision support, and information systems [3]
\end{abstract}

\section{Research Site and DSS}

University of Missouri Healthcare System's (MU) family medicine physicians provide care at nearly 100,000 patient visits per year at eight teaching clinics, including two serving rural mid-Missouri populations. A pilot medical home model was implemented in 2008 in eight Family and Community Medicine clinics through University Physicians. Part of the medical home pilot is the development of a graphic user interface design that was the 2008 winner of the CHIME collaboration award for a medical home clinical information system for the management of diabetes mellitus. Providing quality care for type-2 diabetes requires periodic checks of multiple measures, including laboratory work, medications, physical exam components, and an ophthalmology exam.

The task of the primary care physician or specialist, at any one visit for a patient with type 2 diabetes, involves checking on the status of multiple measures. An evidence-based chronic disease management model was developed and is shown in Figure 1.

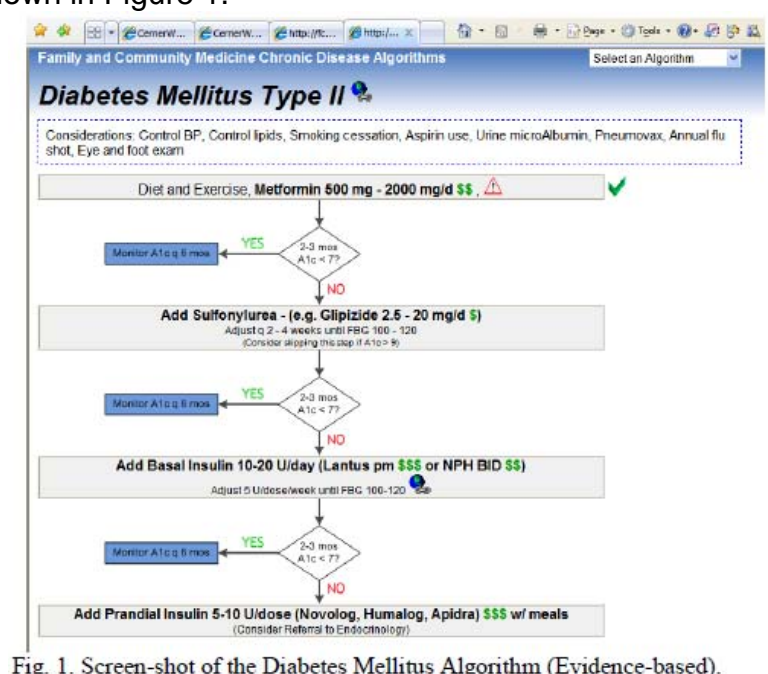

Analysis and Results

\section{We evaluated the efficiency and accuracy of the diabetes} condition summary in comparison to the conventional process of looking through the EMR. Ten family medicine and internal medicine physicians searched for data in the EHR on simulated patients - first using the conventional method, then the condition summary screen. They were recorded with

Morae software and asked to describe their activities with "thinkaloud" interview methods. Figure 2 shows performance by the individual physicians. The mean time to access all data elements using the conventional method was 401 seconds (s.d. 128) compared to 121 seconds (s.d. 40), p<0.001 using the condition summary screen. Considering time on task (that is subtracting time needed to record results for the purposes of this study), time decreased from 5.4 to 1.3 minutes. "Time on Task," Accuracy increased from $94 \%$ to $100 \%$ when using the condition summary. Physicians did not find all data when using the conventional method, with date of last eye and foot exam being the most difficult to extract. A common theme for physicians was, in everyday practice, if they spent too much time searching for a piece of data, they would proceed without it, ask the patient, or order that test again.

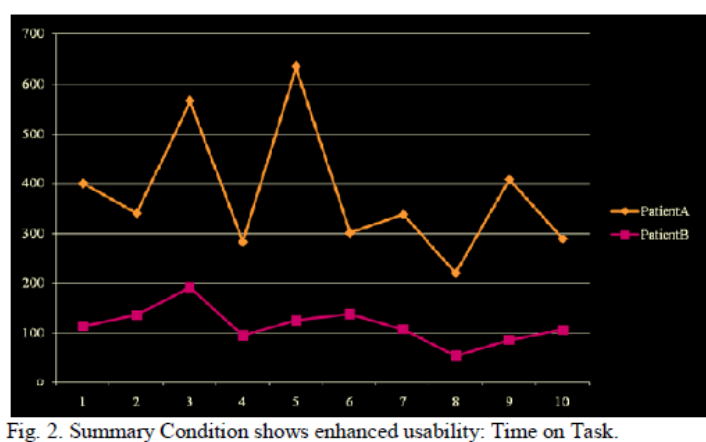

Conclusion and Next Steps

Our preliminary work involved the development of a clinical decision support system for type-2 diabetes. Next steps involve a comprehensive evaluation of the decision support system for its effectiveness and its impact on the overall care delivery process through a simulation model.

The parameters for the simulation model and the evaluation will be built based on data discovery from one hospital system. The study will track these 2,451 patients through a six-month time period of their history in terms of access, utilization of primary care, coordination aspects and their access and utilization of specialists and the hospital. Since the entire system of hospitals and clinics is on an integrated information system, it is easier to track data on these patients across different entities. The degree of level of medical home development within the clinics provides us with two test groups to compare - the first group with a low level medical home capability and only minimal clinical information system capability and, the second with all medical home capabilities.

The study can be extended to include other chronic diseases with the medical home setting.

\section{References}

1. Bodenheimer, T., Wagner, E. H., \& Grumbach, K. (2002). Improving Primary Care for Patients with Chronic Illnesses. JAMA , 288(7): 889893.

2. Wagner, E. H., Austin, B. T., Davis, C., Hindmarsh, M., Schaefer, J., \& Bonomi, A. (2001). Improving Chronic Illness Care: Translating Evidence into Action. Health Affairs , 20: 64-78. 3. ACP. (2006). The Advanced Medical Home: A Patient-centered, Physician-guided Model of Health Care. American College of Physicians. 